\title{
A case of steroid-resistance Hashimoto's encephalopathy presenting with sensorimotor polyneuropathy
}

\author{
Serhat Emeksiz ${ }^{1}$, Nurettin Onur Kutlu ${ }^{1}$, Nuri Alaçakır ${ }^{1}$, Hüseyin Çaksen ${ }^{2}$ \\ ${ }^{1}$ Division of Pediatric Critical Care, ${ }^{2}$ Division of Pediatric Neurology, Department of Pediatrics, Necmettin Erbakan \\ University, Meram Faculty of Medicine, Konya, Turkey \\ E-mail: serhatemeksiz@yahoo.com
}

Received: 13th May 2015, Revised: 26th July 2017, Accepted: 22nd August 2017

\begin{abstract}
SUMMARY: Emeksiz S, Kutlu NO, Alaçakır N, Çaksen H. A case of steroidresistance Hashimoto's encephalopathy presenting with sensorimotor polyneuropathy. Turk J Pediatr 2018; 60: 310-314.

Hashimoto's encephalopathy (HE) is a rare, auto-immune disease characterized by symptoms of acute or subacute encephalopathy associated with increased anti-thyroid antibody levels. The course of most HE cases is relapsing and remitting, which is similar to that of vasculitis and stroke. Steroids are the first line treatment in HE. In steroid non-responders other immunomodulatory therapies or plasmapheresis could be applied. We report a case of steroidresistance $\mathrm{HE}$ with sensorimotor polyneuropathy, as a rare presentation of this disorder. Our case showed that HE may present with sensorimotor polyneuropathy; therefore HE should be considered in the differential diagnoses of polyneuropathy.
\end{abstract}

Key words: steroid-resistance Hashimoto's encephalopathy,sensorimotor polyneuropathy, plasmapheresis, cyclophosphamide, auto-immune disease.

Encephalopathy associated with autoimmune thyroid disease, also called Hashimoto's encephalopathy (HE), is known as a steroidresponsive encephalopathy accompanying high titers of antithyroid antibodies in the absence of central nervous system (CNS) infection, tumor and stroke. ${ }^{1}$ Clinical presentations of HE range from amnestic syndrome, seizures including status epilepticus, ataxia, myoclonus and psychiatric manifestations including depression, mania, psychosis and hallucinations. ${ }^{2}$ Early diagnosis and treatment with immunomodulatory drugs may result in complete recovery. ${ }^{3}$

To the best of our knowledge only a few cases of HE associated with sensorimotor polyneuropathy have been reported in the literature. Herein, we report a case of steroid, immunosuppressive and plasmapheresis resistance HE presenting with sensorimotor polyneuropathy.

\section{Case Report}

A 16-year-old girl was admitted to outpatient clinic of pediatric neurology with tremor and difficulty in walking and speaking. After an uneventful pregnancy she was born at term to healthy, non-consanguineous parents. Her previous medical and family histories were unremarkable. She complained of fatigue, weakness, dizziness, intermittent generalized headache and decline in school performance for the last 15 months, and she had used quetiapine for mood disorder irregularly. She had a 4-month history of edema in lower extremities and tremor inupper extremities. Deterioration of normal movements in the extremities with intermittent choreiform movements had increased, and her speech had declined for the last two weeks.

On physical examination, she was agitated and lethargic. She cooperated poorly during the testing for comprehension, orientation, computation, and memory; however, memory and cognitive impairments were evident. Her initial vital signs were stable, with a body temperature of $36.6^{\circ} \mathrm{C}$, heart rate of 85 beats per minute, respiratory rate of 18 breaths per minute and blood pressure 105/70 $\mathrm{mmHg}$. On neurological examination; Glasgow Coma Score was E4 M6 V3. She had 3/5 muscle strength 
in the upper extremities, $1 / 5$ in the lower extremities. Her pupils were symmetrical and bilaterally reacting normally to light stimulus. There was no facial asymmetry. Deep-tendon reflexes were absentat lower extremities. Plantar responses were flexor, bilaterally. There were no signs of meningeal irritation. She had tremor on upper limbs, edema and limitation of motion on lower limbs. There was no palpable goiter. Other physical examination findings were within normal limits.

The initial laboratory findings were as follows: white blood cell count $16,100 / \mathrm{mm} 3$, hemoglobin $11.3 \mathrm{~g} / \mathrm{dl}$, platelet count $329,000 / \mathrm{mm} 3$ and C-reactive protein $8.14 \mathrm{mg} / \mathrm{dl}$ (normal $5 \mathrm{mg}$ / $\mathrm{dl})$. Routine biochemical analyses of serum, blood gases, electrolytes, blood ammonia, and lactate were innormal range. Thyroid function tests were also within normal limits (free T3 titer was $2.5 \mathrm{pg} / \mathrm{ml}$ (normal 2.5-3.9 pg/ml); free T4 titer was $1.11 \mathrm{ng} / \mathrm{dl}$ (normal 0.6-1.1 $\mathrm{ng} / \mathrm{dl}$ ) and TSH titer was $1.04 \mathrm{IU} / \mathrm{ml}$ (0.36- 5.6 $\mathrm{IU} / \mathrm{ml}$ ). High levels of antithyriod antibodies were noted, with anti-thyroglobulin 546 IU/ $\mathrm{ml}$ (normal 0-40 IU/ml) and anti-thyroidperoxidase (anti-TPO) $1010 \mathrm{U} / \mathrm{ml}$ (normal 0-9 $\mathrm{U} / \mathrm{ml}$ ). Markers of collagen vascular diseases were negative (antinuclear antibody titer, antidouble-stranded DNA, anti- smith antigen, lupic anticoagulant, complement $\mathrm{C} 3-\mathrm{C} 4$, rheumatoid factor). 24-hour provoked urine excretion test for heavy metals (arsenic (As), cadmium (Cd), lead $(\mathrm{Pb})$, and mercury $(\mathrm{Hg})$ ) was negative. Urine toxicology was negative. Vitamin levels were normal. Serum analysis was negative for anti-ganglioside antibodies (anti-GM1, antiGM2) and antibodies against myelin-associated glycoprotein (anti-MAG).Cerebrospinal fluid (CSF) examination was normal. Blood and CSF examination for viral markers and cultures were negative. CSF antithyroid antibodies were negative. Initial chest X-ray was normal. The motor nerve conduction studies at the right median, ulnar and peroneal nerves were not evoked. The results of sensory nerve conduction studies of the right median nerve $(15 \mathrm{~cm}$ proximal to the wrist; amplitude reduction: K75\%) were as follows: Distal conduction velocity $(\mathrm{CV})$ was $50 \mathrm{~m} / \mathrm{s}$, distal latency was $3.04 \mathrm{msn}$ and distal compound muscle action potential (CMAP) amplitude was 8 $\mathrm{mV}$. The results of right ulnar nerve $(12 \mathrm{~cm}$ proximal to the wrist; amplitude reduction:
K58\%): Distal CV was $49.2 \mathrm{~m} / \mathrm{s}$, distal latency was $2.44 \mathrm{msn}$ and distal CMAP amplitude was $6 \mathrm{mV}$. Sural nerves were not evoked. Concentric needle electromyography (CNEMG) examination of right $\mathrm{m}$. abductor digitiminimi, and right $\mathrm{m}$. tibialis anterior showed no voluntary movement. Myopathic motor unit action potentials (MUAP) of m. biceps brachii revealed an increase. These electromyography findings suggested mixed sensorimotor polyneuropathy. Electroencephalography (EEG) showed bilateral diffuse slow wave activity suggestive of encephalopathy. Brain magnetic resonance imaging (MRI) was normal. Clinical, MRI, EEG, electromyography and laboratory findings led to the diagnosis of HE associated with sensorimotor polyneuropathy, and highdose methyl-prednisolone (1 g per day) was administered intravenously on five following days.

Second day of hospitalization, she was intubated due to deterioration of consciousness and GCSless than eight and admitted to the pediatric intensive care units (PICU). Intravenous methylprednisolone therapy was continued. Cefepime and acyclovir were given. On the 5th day of admission she had a seizure, and phenytoin treatment was started. Intravenous methylprednisolone therapy was switched to oral prednisone $(60 \mathrm{mg}$ per day). Cefepime was changed to piperacilintazobactam, teicoplaninand amikacin due to uncontrolled pneumonia. Also alprazolam was started for action tremor and myoclonus in upper extremities. Over the next few days, her encephalopathy state was overtly progressed. Tracheostomy was performed because she was not weaned off ventilator therapy. In the absence of clinical improvement with steroid therapy, plasmapheresis treatment was performed on the 14th day while continuing prednisone therapy. Exchanged plasma volume per treatment was 1.5 times of the total plasma volume (14 exchanges per every other day). Her state of consciousness was partial responded to plasmapheresis, but failed to complete well-being. Two-weeks after plasmapheresis; tremor, myoclonus, and choreoathetotic movements on upper limbs were increased in spite of oral baclofen, primidone and prednisone treatments. On the 12th week of hospitalization, cyclophosphamide was started at the dose of $500 \mathrm{mg} / \mathrm{m}^{2}$ per month in addition 
to oral prednisolone therapy. However, her clinical state did not improve. Because of bone marrow suppression and recurrent infections, the second dose of cyclophosphamide could be given about two months after the first dose of cyclophosphamide.

Due to her clinical condition, she was not sent home. After hospitalization in pediatric neurology clinic about two weeks on homemechanical ventilation, she was re-admitted to PICU because of septic shock. Unfortunately, the patient died due to severe catecholamineresistant shock and multiple organ failure on the 8th day of re-admission at the PICU in spite of antibiotic therapy and renal replacement treatment.

Written consent for publication of this case report, accompanying images and videos was obtained from the parents of the patient.

\section{Discussion}

In the light of our findings, we excluded other differential diagnoses such as CNS infection, acute disseminated encephalomyelitis, intoxications, primary hypoxic injury, prolonged hypoglycemia, degenerative diseases and toxic encephalopathies. We also confirmed our diagnosis, Hashimoto encephalopathy, with elevated serum levels of anti-thyroid antibodies, typical clinical picture and abnormal EEG. Elevated anti-thyroid antibodies are generally considered necessary for diagnosis of HE. The role of antithyroid antibodies in the pathogenesis of HE is still not clear. ${ }^{4}$ However, a recent report has shown that they are capable of binding cerebellar astrocytes suggesting a pathogenic role. ${ }^{5}$ Also, antithyroid antibody levels usually do not correlate with severity of clinical symptoms. ${ }^{6}$ After treatment with steroids or immunosuppressive, antithyroid antibodies titers generally decrease to normal

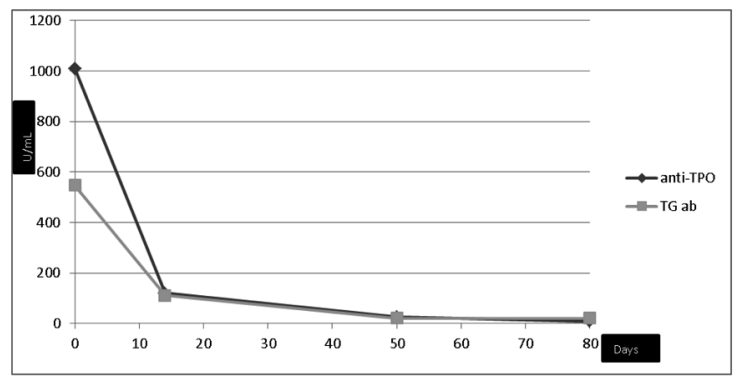

Fig. 1. Antithyroid antibodies levels. levels. ${ }^{7}$ Similarly, theantithyroid antibodies of the present case decreased to normal levels just after the firstplasma exchange and maintained its physiological levels throughout her follow-up as depicted in Figure 1. But unfortunately, clinical improvement was not seen in spite of whole immunosuppressive and plasmapheresis treatments. Thyroid status varies among patients with reported $\mathrm{HE}$, ranging from overt hypothyroidism to overt hyperthyroidism. In systematic reviews, 23 to 35 percent of patients with HE have subclinical hypothyroidism and 17 to 20 percent have overt hypothyroidism. ${ }^{8}$ About 7 percent are hyperthyroid; the remainder are euthyroid. Our patient was euthyroid similar to the literature.

Two-thirds of patients may experience focal or generalized tonic-clonic seizures, and $12 \%$ may present with status epilepticus. ${ }^{9}$ Also, hyperreflexia and other pyramidal tract signs can be seen in $85 \%$ of patients; and psychosis, visual hallucinations and paranoid delusions have been reported in $25 \%$ to $36 \%$ of patients. ${ }^{9}$ Myoclonus and tremor, often of the bilateral upper extremities and palatal myorhythmia, are the most frequently observed involuntary movements in HE. ${ }^{5}$ Our patient'sinitial presentation was psychosis which began 15 months before admission, and quetiapine was irregularly used.

As, an autoimmune basis is suggested in $\mathrm{HE}$, various immunosuppressive treatments have been used in this condition. ${ }^{8}$ Patients respond dramatically to steroid therapy. Initial dose varies between $50 \mathrm{mg}$ and $150 \mathrm{mg}$ of oral prednisolone daily, slowly tapered over weeks to months, depending on clinical course of the disease. High dose intravenous methylprednisolone ( $1 \mathrm{~g} /$ day) may also be given for initial 3 to 7 days, followed by oral prednisolone therapy. ${ }^{10}$ Around $2-5 \%$ of patients may not respond to steroids, in whom immunoglobulins, plasmapheresis, azathioprine or cyclophosphamide can be used with good results. ${ }^{9-11}$ In our case, intravenous methylprednisolone therapy, ( $1 \mathrm{~g}$ per day for 5 days) and then oral prednisolone $(60 \mathrm{mg} /$ day) were used. Because of no response to steroid therapy, plasmapheresis and cyclophosphamide were used. However, no adequate clinical response was received.

It's not always easy to pinpoint the cause of 
polyneuropathy because a number of factors can cause neuropathies. These factors include: heavy metal toxicity, chemotherapy, viral or bacterial infections, trauma, tumors, kidney disease, liver disease, connective tissue disorders, an underactive thyroid (hypothyroidism) and autoimmune diseases (Sjogren's syndrome, lupus, rheumatoid arthritis, Guillain-Barre syndrome). ${ }^{12}$ A comorbidity of $\mathrm{HE}$ and sensorimotor polyneuropathy has been rarely reported in the literature. ${ }^{13,14}$ In the adult literature, isolated reduction of sensory nerve action potential amplitudes was reported in a patient with a diagnosis of HE. ${ }^{15}$ In that case the authors concluded that there was an involvement of the dorsal root ganglia, namely a sensory ganglioneuropathy. Interestingly, there was also a report on an adult patient with Hashimoto's thyroiditis who developed isolated peripheral nervous system involvement consisting of chronic inflammatory demyelinating polyneuropathy; in that case neurophysiological tests indicated prolonged sensory and motor terminal latencies, slowing of conduction block in nerve segments and absent F-waves. ${ }^{13}$ To the best our knowledge, peripheral nerve involvement in HE was reported in only one case in the pediatric literature. Salpietro et al. ${ }^{16}$ reported a 13 -year-old girl with HE who developed distal neuropathy. Her electromyography and nerve conduction velocity tests showed prolonged sensory latencies and reduced amplitude of action potentials in the upper and lower limbs segments with absent F-waves in the popliteal regions and the ankles bilaterally; motor conduction velocity was normal, indicating distal neuropathy (likely a polyneuropathy). The patient was treated with i.v. methylprednisolone $(20 \mathrm{mg} / \mathrm{kg})$ coupled with i.v. immunglobulin $(360 \mathrm{mg} / \mathrm{kg})$ for 3 days. Our patient's EMG findings suggested mixed sensorimotor polyneuropathy but she did not response to steroid therapy, plasmapheresis and cyclophosphamide. We consider that there is a relationship between $\mathrm{HE}$ and polyneuropathy reflecting a peripheral manifestation of the underlying immunological mechanism.

In conclusion, $\mathrm{HE}$ is a rare condition in childhood but should always be considered in children with psychiatric symptoms with unexplained clinical presentations, seizure, confusion, and behavioral changes. As HE usually responds to corticosteroid therapy well, corticosteroids are the preferred drugs for its treatment, whereas other immunosuppressants have also been reported to treat HE successfully. Most patients have a good prognosis unless there is a delay in diagnosis and treatment. Our case showed that HE may be presented with sensorimotor polyneuropathy; therefore $\mathrm{HE}$ should be considered in differential diagnoses of sensorimotor polyneuropathy.

\section{REFERENCES}

1. Yu HJ, Lee J, Seo DW, Lee M. Clinical manifestations and treatment response of steroid in pediatric Hashimoto encephalopathy. J Child Neurol 2014; 29: 938-942.

2. Wang J, Zhang J, Xu L, Shi Y, Wu X, Guo Q. Cognitive impairments in Hashimoto's encephalopathy: A casecontrol study. PLoS One 2013; 8: e55758.

3. Ghoreishi E, Shahidi GA, Rohani M, Nabavi M, Aghaei M, Akhoundi FH. Palatal-Myoclonus as a presentation of Hashimoto Encephalopathy: An interesting case report. Iran J Psychiatry 2013; 8: 149-151.

4. Huang $\mathrm{X}, \mathrm{Yu} \mathrm{Y}$, Zhang $\mathrm{H}$, et al. Hashimoto encephalopathy associated with hyperthyroidism: A case report. Exp Ther Med 2014; 8: 515-518.

5. Tang Y, Xing Y, Lin MT, Zhang J, Jia J. Hashimoto's encephalopathy cases: Chinese experience. BMC Neurol 2012; 12: 60 .

6. Santra G, De D, Phaujdar S, Rudra A, Dutta PS. Hashimoto's encephalopathy. J Assoc Physicians India 2012; 60: 48-51.

7. Singh H, Ray S, Agarwal S, Verma RP, Talapatra P, Gupta V. Spectroscopic correlation and role of Azathioprine in long-term remission in patients of Hashimoto encephalopathy. Ann Indian Acad Neurol 2013; 16: 443-446.

8. Chong JY, Rowland LP, Utiger RD. Hashimoto encephalopathy: Syndrome or myth? Arch Neurol 2003; 60: 164-171.

9. Philip R, Saran S, Gutch M, Gupta K. An unusual presentation of Hashimoto's encephalopathy. Indian J Endocrinol Metab 2014; 18: 113-115.

10. Shaw PJ, Walls TJ, Newman PK, Cleland PG, Cartlidge NE. Hashimoto's encephalopathy: A steroid-responsive disorder associated with high anti-thyroid antibody titers--report of 5 cases. Neurology 1991; 41( 2 Pt 1): $228-233$.

11. Nieuwenhuis L, Santens P, Vanwalleghem P, Boon P Subacute Hashimoto's encephalopathy, treated with plasmapheresis. Acta Neurol Belg 2004; 104: 80-83.

12. Menkes JH. Textbook of Child Neurology (7th ed). Philadelphia: Lea and Febiger, 2005: 127-128.

13. Raghavendra S, Sanjay S, Somashekar R, Ashalatha R, Shankar SK. CIDP, Hashimoto's thyroiditis and nephropathy: Autoimmune syndrome complex? Can J Neurol Sci 2009; 36: 382-384. 
14. Sheng B, Lau KK, Li HL, Cheng LF. A case of Hashimoto's encephalopathy with demyelinating peripheral neuropathy. Eur Neurol 2005; 53: 84-85.

15. Bairactaris C, Stouraitis G, Papalias E, Fakas N, Papatheodorou G, Gourtzelidis P. Early neurophysiological evolution of chronic inflammatory demyelinating polyneuropathy in a patient with Hashimoto's thyroiditis. Muscle Nerve 2008; 38: 15181522 .
16. Salpietro V, Mankad K, Polizzi A, et al. Pediatric Hashimoto's encephalopathy with peripheral nervous system involvement. Pediatr Int 2014; 56: 413-416. 